\title{
Changing the obesogenic environment to improve cardiometabolic health in residential patients with a severe mental illness: cluster randomised controlled trial*
}

Anne Looijmans, Annemarie P. M. Stiekema, Richard Bruggeman, Lisette van der Meer, Ronald P. Stolk, Robert A. Schoevers, Frederike Jörg** and Eva Corpeleijn**

\section{Background}

For patients with severe mental illness (SMI) in residential facilities, adopting a healthy lifestyle is hampered by the obesity promoting (obesogenic) environment.

\begin{abstract}
Aims
To determine the effectiveness of a 12-month lifestyle intervention addressing the obesogenic environment with respect to diet and physical activity to improve waist circumference and cardiometabolic risk factors $v$. care as usual (Dutch Trial Registry: NTR2720).
\end{abstract}

\section{Method}

In a multisite cluster randomised controlled pragmatic trial, 29 care teams were randomised into 15 intervention (365 patients) and 14 control teams (371 patients). Intervention staff were trained to improve the obesogenic environment.

\section{Results}

Waist circumference decreased $1.51 \mathrm{~cm}(95 \% \mathrm{Cl}-2.99$ to -0.04 ) in the intervention $v$. control group after 3 months and metabolic syndrome $z$-score decreased 0.22 s.d. $(95 \% \mathrm{Cl}$ -0.38 to -0.06 ). After 12 months, the decrease in waist circumference was no longer statistically significantly different $(-1.28 \mathrm{~cm}, 95 \% \mathrm{Cl}-2.79$ to $0.23, P=0.097)$.

\section{Conclusions}

Targeting the obesogenic environment of residential patients with SMI has the potential to facilitate reduction of abdominal adiposity and cardiometabolic risk, but maintaining initial reductions over the longer term remains challenging.

\section{Declaration of interest}

None.

\section{Copyright and usage}

(c) The Royal College of Psychiatrists 2017.
Patients with a severe mental illness (SMI), mostly schizophrenia, other psychotic disorders, major depression or bipolar disorder, have almost twice the normal risk of premature death from cardiovascular disease, ${ }^{1}$ are more likely to have metabolic syndrome, ${ }^{2}$ and have a life expectancy that is shortened by up to 30 years compared with the general population. ${ }^{3}$ The increased mortality risk is associated with side-effects of antipsychotic medication as well as unhealthy but modifiable lifestyle behaviours. ${ }^{4}$

Lifestyle interventions in patients with SMI have previously been shown to reduce body weight ${ }^{5}$ and waist circumference, and to improve cardiometabolic risk factors such as serum triglyceride levels, fasting glucose and insulin concentrations. ${ }^{6,7}$ These studies included mostly out-patients ${ }^{8,9}$ or individuals with a first-episode of schizophrenia, ${ }^{10,11}$ whereas studies in patients with SMI admitted to sheltered or clinical care facilities are scarce. In addition, sustainability of effects is questionable.

Most lifestyle interventions are designed to stimulate individuals to change their diet and physical activity behaviour and involve counselling, goal-setting and weight monitoring. The challenge of these programmes is that they are highly dependent on individual patients' interests, motivation and capacities, which are reduced in people with SMI because of negative symptoms and cognitive deficits. ${ }^{12}$ In residential facilities, the setting is important as well since facilities are often characterised by an 'obesogenic' environment as a result of an abundant provision of unhealthy food products and a lack of daily activities. ${ }^{13,14}$ An approach focusing on the obesogenic environment, 'making the healthy choice the

*This work was presented, in part, at the 25th European Congress of Psychiatry, Florence, 1-4 April 2017

**These authors contributed equally to the work as senior authors easy choice' by educating staff how to change daily practice with regard to healthy nutrition and physical activities in the facility, may lead to sustainable changes for all residential patients with SMI. Two studies have addressed the obesogenic environment of residential patients with SMI by modifying food delivery ${ }^{15}$ or adjusting the offered food combined with nutritional counselling and exercise sessions, ${ }^{16}$ and reported promising improvements in patients' somatic health. However, these studies lacked a control group ${ }^{15}$ or had a small sample size. ${ }^{16}$ Another approach that may work well for the SMI population is the 'small change approach'. This approach aims for modest lifestyle changes leading to modest, but sustainable weight loss in the long term. ${ }^{17}$

We developed the Effectiveness of Lifestyle Interventions in PSychiatry (ELIPS) trial. ${ }^{18}$ In this trial, we designed a lifestyle intervention to improve cardiometabolic health of patients with SMI living in residential facilities by stimulating a healthy lifestyle via small but sustainable changes in the obesogenic environment. The ELIPS trial is a pragmatic randomised controlled trial (RCT), designed to offer tailored, scalable and implementable interventions. ${ }^{19}$ This means that already in the trial phase, the intervention was aimed at and implemented by regular staff members in daily care. We expected stable or improved cardiometabolic health in the intervention group compared with deteriorated cardiometabolic health in the control group. In addition, we explored whether the intervention effect depends on gender, age and type of facility.

\section{Method}

The ELIPS study protocol was published ${ }^{18}$ and will be briefly explained below. The Medical Ethical Committee for Research in Mental Health Care (Metigg) concluded that the study protocol 
and use of anonymised data from Routine Outcome Monitoring (ROM; below) was in accordance with the Declaration of Helsinki and (inter)national regulations, and that the study did not fall under the scope of the Medical Research Involving Human Subjects Act, thereby waiving informed consent. The trial was registered in the Dutch Trial Registry (NTR2720, www.trialregister.nl).

\section{Participants and recruitment}

Patients with SMI from all sheltered and long-term clinical care teams ( $n=29$ teams, $20-65$ patients per team) of two mental health organisations in The Netherlands were included in the study from September 2010 until December 2011 if they participated in the annual ROM (below) (Fig. 1). Long-term clinical care facilities deliver direct, all-day intensive professional care. Sheltered facilities provide supported living, a combination of housing and services in the community. Exclusion criteria were age below
18, pregnancy, Korsakoff syndrome or inability to participate in tests. In total 240 patients per arm were needed to detect a clinical relevant change of $-5 \%$ in waist circumference $(\alpha=0.05$, power 0.90), taking into account estimated ROM screenings performed in a well-established infrastructure.

\section{Intervention}

The ELIPS intervention was directed at nursing teams and addressed the obesogenic environment of patients; see ELIPS study protocol $^{18}$ for examples from practice. The intervention consisted of a preparation, implementation and monitoring phase. In the 1-month preparation phase, lifestyle coaches introduced themselves to staff and patients, screened the environment and teams' daily routines, and listed patients' and teams' preferences and sites' logistic possibilities. Lifestyle coaches created a team-tailored lifestyle plan based on listed preferences and possibilities and four pre-established ELIPS lifestyle goals:

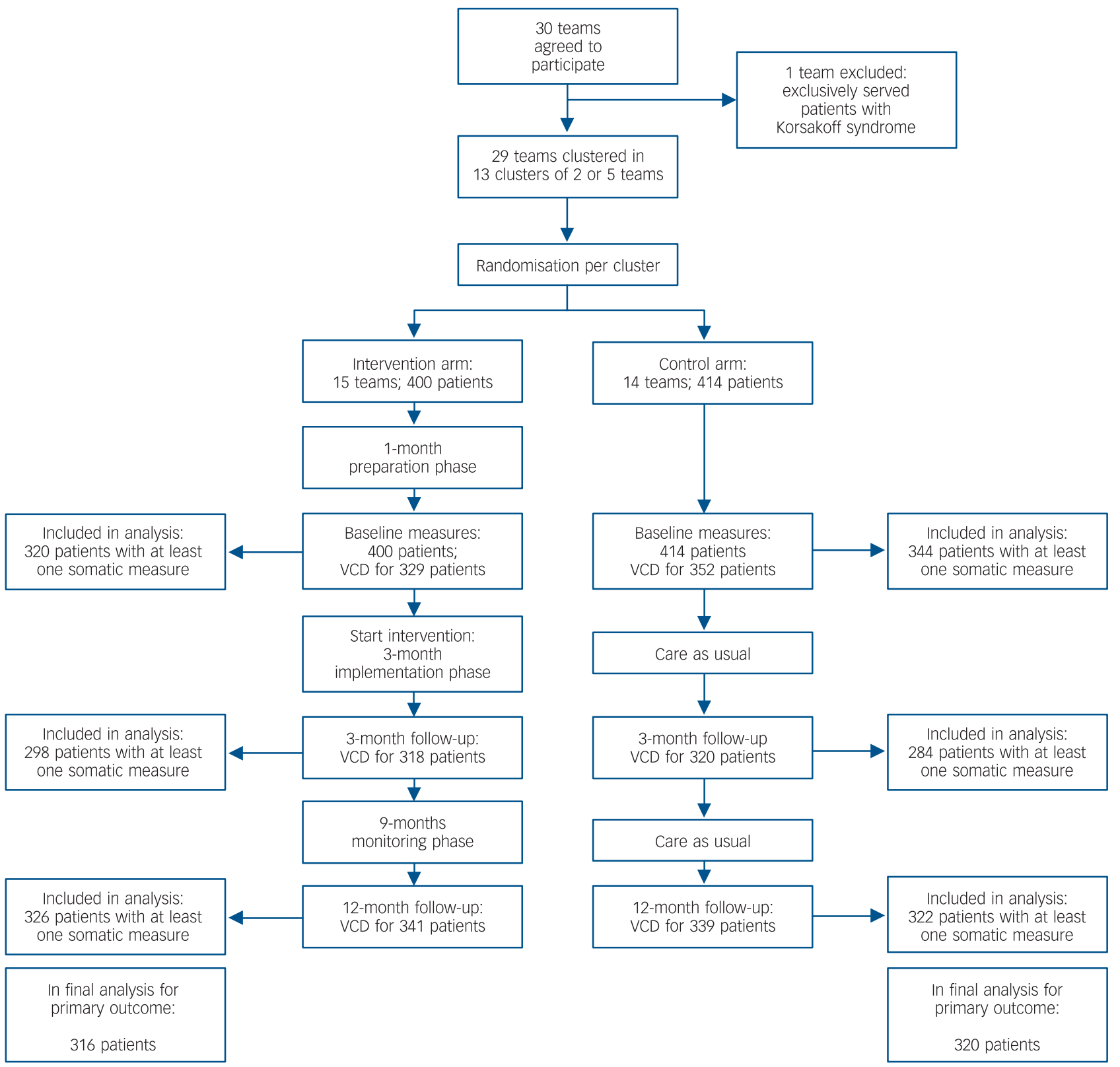

Fig 1 Flow chart of patients in the Effectiveness of Lifestyle Interventions in PSychiatry (ELIPS) trial. 
(a) to stimulate physical activity; (b) to increase supply/availability of healthy food products; (c) to organise at least one activity per week focused on a healthy diet; and (d) to improve the obesogenic environment at an organisational level. In the 3-months implementation phase, lifestyle coaches implemented the planned ELIPS lifestyle activities as described in the team-tailored lifestyle plan. Lifestyle coaches first demonstrated activities to staff, then carried out the activities together with staff and finally supervised staff while they carried out the activities. Lifestyle coaches trained teams to create a healthy environment and stimulate healthy behaviours in patients. At the end of the implementation phase, teams set goals to achieve in the 9-month monitoring phase. In the monitoring phase, a lifestyle coach visited all intervention teams twice and discussed with the team and team leader whether goals were achieved, which barriers in achieving the goals were encountered and discussed options to tackle these barriers. Also, the researchers organised one benchmark meeting for all intervention team leaders where difficulties in achieving team goals were discussed and tips, tricks and successful examples were shared.

Lifestyle coaches were trained for 2 days about the ELIPS lifestyle programme, motivational interviewing techniques and the patient population. Lifestyle coaches were fulfilling the final of 4 years of education to become professional lifestyle coaches. Because lifestyle coaches were still in training, each team had two lifestyle coaches at its disposal, who were appointed by the research team. Per week, lifestyle coaches spent on average $8 \mathrm{~h}$ on activities with patients ( 6 contact hours and about $2 \mathrm{~h}$ preparation time) and $8 \mathrm{~h}$ on training of staff and organisational aspects, such as developing information materials, meetings with staff and project management.

Patients in the control condition received care as usual. Lifestyle initiatives in control teams were documented. Randomisation was performed at team level using a randomised block design with 12 clusters of two comparable teams and 1 cluster of five comparable teams based on mental healthcare organisation, type of facility, case-load size and location (urban or rural). Teams were randomised with 55 into the intervention or control arm with computerised random number generator by a non-participating research nurse.

\section{Measurements and outcomes}

Primary outcome was waist circumference at 3 and 12 months after baseline. Secondary outcomes were body mass index (BMI; $\mathrm{kg} / \mathrm{m}^{2}$ ) and metabolic syndrome $z$-score. Information on age, gender, diagnosis and medication use was derived from patient records. Physical health data were collected by trained nurses in annual ROM screenings, part of the ongoing PHAMOUS (Pharmacotherapy Outcome and Monitoring Survey) cohort. ${ }^{20}$ Annual ROM screenings are standard care in both organisations and were rescheduled 1-2 weeks before the start of the intervention (baseline measure) and 3 and 12 months thereafter (follow-up measurements). Patients received a small fee for the additional 3-months ROM screening ( $€ 5.00 / \$ 5.38)$. ROM nurses were masked to intervention allocation. Waist circumference was measured twice in the standing position at the end of an expiration using a flexible non-stretching tape halfway between iliac crest and lowest rib. Weight was measured by calibrated scales (Seca, model 813, Hamburg, Germany) in light clothing without shoes or jackets. Height was measured without shoes. Systolic and diastolic blood pressure (BP) were measured using an automated blood pressure monitor (BOSO medicus control, Jungingen, Germany) in the sitting position after $5 \mathrm{~min}$ rest. Patients visited a (hospital) laboratory to collect a fasting blood sample for levels of lipids (total cholesterol, low-density lipoprotein
(LDL)-cholesterol, high-density lipoprotein (HDL)-cholesterol and triglycerides) and glucose metabolism (glucose, haemoglobin $\mathrm{A}_{1 \mathrm{c}}(\mathrm{HbAlc})$ ). If not fasting, this was routinely indicated on the form by the nurse.

The metabolic syndrome was defined as the presence of three or more of the following criteria: ${ }^{21}$ (a) waist circumference $\geqslant 88$ / $102 \mathrm{~cm}$ (female/male); (b) systolic BP $\geqslant 130$ and/or diastolic $\mathrm{BP} \geqslant 85 \mathrm{mmHg}$ or receiving antihypertensive medication; (c) HDL-cholesterol $<1.03 / 1.3 \mathrm{mmol} / \mathrm{L}$ (female/male; divide by 0.0259 to convert to $\mathrm{mg} / \mathrm{dL}$ ) or receiving lipid-lowering medication; (d) triglycerides $\geqslant 1.7 \mathrm{mmol} / \mathrm{L}$ (divide by 0.0113 to convert to $\mathrm{mg} / \mathrm{dL}$ ) or receiving lipid-lowering medication; and (e) fasting glucose $\geqslant 6.1 \mathrm{mmol} / \mathrm{L}$ (divide by 0.0555 to convert to $\mathrm{mg} / \mathrm{dL}),{ }^{22}$ receiving antihyperglycemic medication or reporting a diagnosis for diabetes. When fasting glucose levels were not available (baseline: $46 \%, n=342 ; 3$ months: $53 \%, n=392 ; 12$ months: $46 \%, n=342$ ), patients were considered to fulfil the glucose-risk criterion if they reported having diabetes $(9.6 \%$, $n=71)$ or if $\mathrm{HbAlc} \geqslant 6.0 \%{ }^{23}$ The individual components were standardised into $z$-scores (with HDL-cholesterol $z$-score multiplied by -1$)^{24,25}$ and the sum divided by 5 was used as a continuous variable for the degree of metabolic syndrome. BP was standardised using mean arterial pressure (MAP). Antipsychotic medication was categorised in three groups according to the strength of the side-effects on cardiometabolic health (none, mild or strong) based on the literature (see online Table DS1). ${ }^{26,27}$

\section{Statistical analyses}

Data were analysed according to the intention-to-treat principle using SPSS version 22. A $P<0.05$ was considered statistically significant. Results are reported as mean (95\% confidence interval). Differences in frequency distributions were tested with chi-square or Mann-Whitney $U$-tests. For testing main differences between intervention and control, a likelihood-based general linear mixed model was applied, using a subject-specific model to adjust for clustering of patients within teams using an 'unstructured' error structure, and controlling for the block design. For all analyses, the outcomes over time per patient formed the first level of the model, the patient formed the second level, and team formed the third level and cluster as random factor. Since it is possible that intervention effects on somatic outcomes differ between implementation (first 3 months) and monitoring phase ( 9 months thereafter), time was coded as two dummy variables. Group (intervention or control), time, and group $\times$ time interactions were entered in the model as fixed factors with adjustment for age, gender, type of facility and antipsychotic medication. As a secondary analysis, clinically relevant change was studied, defined as a change of at least $5 \%$ in waist circumference. Finally, we studied the intervention effect within prespecified subgroups (gender, age groups and type of facility).

\section{Results}

The 29 teams were randomised into 14 control and 15 intervention teams, resulting in 814 eligible patients (Fig. 1). Of these, $736(90 \%)$ had at least one physical measurement at baseline or at 12 months and were included in the analyses. The majority of patients were men $(63.2 \%)$, the mean age was 48.3 years (s.d. $=12.6)$ ranging from 20 to 85 years (Table 1). Most patients were overweight or obese $(65.9 \%)$ and $58.4 \%$ met the criteria for metabolic syndrome (Table 2). In the intervention group, $46 \%$ of the patients lived in long-term clinical facilities compared with $36 \%$ in the control group $(P<0.01)$. This yielded a significantly higher age and more psychotic disorders in the intervention group. 


\begin{tabular}{|c|c|c|c|c|c|}
\hline & $n$ & Total & Intervention group & Control group & $P$ \\
\hline Teams, $n$ & 29 & & 15 & 14 & \\
\hline Patients, $n$ & 736 & & 365 & 371 & \\
\hline Age, years: mean (s.d.) & 736 & $48.3(12.6)$ & $49.3(12.0)$ & $47.2(13.2)$ & 0.03 \\
\hline Male gender, $n(\%)$ & 736 & $465(63.2)$ & $236(64.7)$ & $229(61.7)$ & 0.41 \\
\hline Housing, $n$ & 736 & & & & \\
\hline Sheltered living, teams (patients) & & $18(434)$ & $9(196)$ & $9(238)$ & 0.004 \\
\hline Long-term clinical facilities, teams (patients) & & $11(302)$ & $6(169)$ & $5(133)$ & \\
\hline
\end{tabular}

After 3 months of lifestyle intervention, the intervention group showed a significant decrease in waist circumference of $1.51 \mathrm{~cm}(95 \%$ CI -2.99 to -0.04$)$ compared with the control group (Table 3). After 12 months, the waist circumference in the intervention group remained lower than in the control group ( $1.28 \mathrm{~cm}-2.79$ to 0.23 ) although no longer statistically significant. Metabolic syndrome $z$-score decreased by $0.22 \mathrm{~s}$.d. (95\% CI -0.38 to -0.06$)$ in the intervention compared with the control group after 3 months, of which most of the effect was attributable to a significant decrease of $0.48 \mathrm{s.d}$. in glucose $z$-score $(95 \% \mathrm{CI}-0.87$ to -0.09$)$ and of 0.09 s.d. in waist circumference $z$-score $(95 \% \mathrm{CI}-0.18$ to -0.01$)$ in the intervention group. The effect on metabolic syndrome $z$-score was not sustained after 12 months. We found no intervention effects on BMI. In general, changes in waist circumference and BMI over time varied widely between teams in both the intervention and control group (Fig. 2).

In the intervention group, $20.1 \%$ of the participants had a clinically relevant improvement ( $\geqslant-5 \%$ waist circumference) and $20.6 \%$ had a clinically relevant deterioration $(\geqslant+5 \%$ waist

\begin{tabular}{|c|c|c|c|c|c|}
\hline & $n$ & Total & Intervention group & Control group & $P$ \\
\hline $\begin{array}{l}\text { Body mass index (BMI), } \mathrm{kg} / \mathrm{m}^{2} \text { : mean (s.d.) } \\
\text { Normal (BMI <25), } n(\%) \\
\text { Overweight (BMI 25-29), } n(\%) \\
\text { Obese I (BMI 30-34), } n(\%) \\
\text { Obese II (BMI 35-39), } n(\%) \\
\text { Obese III (BMI } \geqslant 40), n(\%)\end{array}$ & 616 & $\begin{array}{c}28.0(6.3) \\
210(34.1) \\
210(34.1) \\
117(19.0) \\
50(8.1) \\
29(4.7)\end{array}$ & $\begin{array}{l}27.8(6.3) \\
108(36.6) \\
100(33.9) \\
48(16.3) \\
27(9.2) \\
12(4.1)\end{array}$ & $\begin{array}{l}28.3(6.2) \\
102(31.8) \\
110(34.3) \\
69(21.5) \\
23(7.2) \\
17(5.3)\end{array}$ & 0.27 \\
\hline \multicolumn{6}{|l|}{ Waist circumference, $\mathrm{cm}$ : mean (s.d.) } \\
\hline $\begin{array}{l}\text { Men } \\
\text { Women }\end{array}$ & $\begin{array}{l}350 \\
208\end{array}$ & $\begin{array}{l}104.4(16.1) \\
103.0(17.0)\end{array}$ & $\begin{array}{l}105.6(15.4) \\
104.0(18.7)\end{array}$ & $\begin{array}{l}103.1(16.8) \\
102.2(15.5)\end{array}$ & $\begin{array}{l}0.14 \\
0.45\end{array}$ \\
\hline Metabolic syndrome, $n(\%)$ & 387 & $226(58.4)$ & $120(58.5)$ & $106(58.2)$ & 0.95 \\
\hline Metabolic syndrome $z$-score, mean (s.d. $)^{b}$ & 387 & $0.45(1.02)$ & $0.48(1.05)$ & $0.42(0.98)$ & 0.59 \\
\hline $\begin{array}{l}\text { Blood pressure (BP), mmHg: } n \\
\text { Systolic BP, mean (s.d.) } \\
\text { Diastolic BP, mean (s.d.) } \\
\text { Use of BP-lowering medication, } n(\%)\end{array}$ & 612 & $\begin{array}{l}129.8(18.8) \\
84.2(12.1) \\
137(21.2)\end{array}$ & $\begin{array}{l}129.9(19.3) \\
84.6(12.8) \\
79(25.2)\end{array}$ & $\begin{array}{c}129.8(18.3) \\
83.8(11.4) \\
58(17.5)\end{array}$ & $\begin{array}{l}0.97 \\
0.43 \\
\mathbf{0 . 0 2}\end{array}$ \\
\hline $\begin{array}{l}\text { Lipids } \\
\text { Total cholesterol, mmol/L: mean (s.d.) } \\
\text { HDL-cholesterol, mmol/L: mean (s.d.) }\end{array}$ & 477 & $5.19(1.13)$ & $5.10(1.16)$ & $5.29(1.08)$ & 0.07 \\
\hline $\begin{array}{l}\text { Men } \\
\text { Women }\end{array}$ & $\begin{array}{l}293 \\
182\end{array}$ & $\begin{array}{l}1.10(0.33) \\
1.33(0.41)\end{array}$ & $\begin{array}{l}1.08(0.32) \\
1.38(0.39)\end{array}$ & $\begin{array}{l}1.12(0.33) \\
1.27(0.43)\end{array}$ & $\begin{array}{l}0.38 \\
0.06\end{array}$ \\
\hline LDL-cholesterol, mmol/L: mean (s.d.) & 461 & $3.19(1.01)$ & $3.07(1.02)$ & $3.33(0.98)$ & 0.005 \\
\hline Triglycerides, mmol/L: median (25-75th percentile) & 475 & $1.67(1.12-2.42)$ & $1.65(1.08-2.35)$ & $1.69(1.14-2.50)$ & 0.58 \\
\hline \multicolumn{6}{|l|}{ Glucose metabolism } \\
\hline $\begin{array}{l}\text { Fasting glucose, mmol/L: median (25-75th percentile), } \\
\text { HbA1c, \%: median (25-75th percentile) } \\
\text { Use of glucose-lowering medication, } n(\%)\end{array}$ & $\begin{array}{l}394 \\
301 \\
646\end{array}$ & $\begin{array}{c}5.60(5.10-6.40) \\
5.60(5.25-6.00) \\
104(16.1)\end{array}$ & $\begin{array}{c}5.60(5.20-6.30) \\
5.70(5.30-6.00) \\
51(16.2)\end{array}$ & $\begin{array}{c}5.60(5.08-6.40) \\
5.50(5.10-5.90) \\
53(16.0)\end{array}$ & $\begin{array}{c}0.34 \\
\mathbf{0 . 0 0 3} \\
0.92\end{array}$ \\
\hline \multicolumn{5}{|l|}{ Psychiatric diagnosis, $n$ (\%) } & \\
\hline Psychotic disorder & & $534(72.6)$ & $277(75.9)$ & $257(69.3)$ & 0.04 \\
\hline Mood disorder & & $76(10.3)$ & $37(10.1)$ & $39(10.5)$ & 0.87 \\
\hline Personality disorder & & $238(32.3)$ & $105(28.8)$ & $133(35.8)$ & 0.04 \\
\hline Psychiatric comorbidity, ${ }^{c} n(\%)$ & & $179(24.3)$ & $79(21.6)$ & $100(27.0)$ & 0.09 \\
\hline Use of antipsychotics, $n$ (\%) & 646 & $581(89.9)$ & $288(91.7)$ & $293(88.3)$ & 0.14 \\
\hline Antipsychotic metabolic side-effect, $n(\%)$ & 581 & & & & 0.77 \\
\hline $\begin{array}{l}\text { None } \\
\text { Mild }\end{array}$ & & $\begin{array}{c}57(9.8) \\
197(33.9)\end{array}$ & $\begin{array}{c}30(10.4) \\
100(34.7)\end{array}$ & $\begin{array}{l}27(9.2) \\
97(33.1)\end{array}$ & \\
\hline Strong & & $327(56.3)$ & $158(54.9)$ & $169(57.7)$ & \\
\hline
\end{tabular}

a. SI unit conversion factors: to convert total cholesterol, high-density lipoprotein (HDL)-cholesterol and low-density lipoprotein (LDL)-cholesterol to $\mathrm{mg} / \mathrm{dL}$, divide values by 0.0259 ; to convert triglycerides to $\mathrm{mg} / \mathrm{dL}$, divide values by 0.0113 ; to convert fasting glucose to $\mathrm{mg} / \mathrm{dL}$, divide values by 0.0555 .

$\mathrm{b}$. The means and standard deviations of the patients ranging within healthy reference values were used to standardise $\mathrm{HDL}$-cholesterol $(1.1-2.0 \mathrm{mmol} / \mathrm{L}$ in women and

$0.9-1.7 \mathrm{mmol} / \mathrm{L}$ in $\mathrm{men})$, triglycerides $(\leqslant 2.2 \mathrm{mmol} / \mathrm{L})$ and fasting glucose $(\leqslant 7.1 \mathrm{mmol} / \mathrm{L})$ or haemoglobin $\mathrm{A}_{1 \mathrm{c}}(\mathrm{HbA} 1 \mathrm{C})(<8.0 \%)$.

c. Two or more of the defined diagnoses. 


\begin{tabular}{|c|c|c|c|c|c|c|}
\hline & \multicolumn{2}{|c|}{ Waist circumference $(n=636)$} & \multicolumn{2}{|c|}{ Body mass index $(n=654)$} & \multicolumn{2}{|c|}{ Metabolic syndrome $z$-score $(n=512)$} \\
\hline & $\beta(95 \% \mathrm{Cl})$ & $P$ & $\beta(95 \% \mathrm{Cl})$ & $P$ & $\beta(95 \% \mathrm{Cl})$ & $P$ \\
\hline \multicolumn{7}{|l|}{ Intervention effect ${ }^{\mathrm{a}}$} \\
\hline 3 months $^{b}$ & $-1.51(-2.99$ to -0.04$)$ & 0.04 & $-0.13(-0.49$ to 0.23$)$ & 0.44 & $-0.22(-0.38$ to -0.06$)$ & 0.008 \\
\hline 12 months $^{b}$ & $-1.28(-2.79$ to 0.23$)$ & 0.10 & $0.34(-0.12$ to 0.79$)$ & 0.14 & $-0.00(-0.16$ to 0.16$)$ & 0.99 \\
\hline $\begin{array}{l}\text { Group difference } \\
\text { (intervention } v \text {. control) }\end{array}$ & $0.44(-2.22$ to 3.09$)$ & 0.75 & $-0.60(-1.56$ to 0.36$)$ & 0.22 & $-0.04(-0.22$ to 0.15$)$ & 0.70 \\
\hline \multicolumn{7}{|l|}{ Time effect only } \\
\hline 3 months & $1.11(0.05$ to 2.16$)$ & 0.04 & $0.10(-0.15$ to 0.36$)$ & 0.40 & $0.13(0.01$ to 0.26$)$ & 0.03 \\
\hline 12 months & $0.75(-0.31$ to 1.80$)$ & 0.17 & $-0.04(-0.36$ to 0.29$)$ & 0.82 & $0.01(-0.10$ to 0.12$)$ & 0.89 \\
\hline Age & $0.13(0.02$ to 0.24$)$ & 0.02 & $-0.02(-0.06$ to 0.02$)$ & 0.31 & $0.00(-0.00$ to 0.01$)$ & 0.51 \\
\hline Male gender & $0.44(-2.15$ to 3.03$)$ & 0.74 & $-2.81(-3.79$ to -1.83$)$ & 0.001 & 0.26 (0.08 to 0.44$)$ & 0.005 \\
\hline Sheltered facility & $0.76(-4.67$ to 6.18$)$ & 0.76 & $0.31(-1.13$ to 1.74$)$ & 0.67 & $-0.13(-0.33$ to 0.08$)$ & 0.20 \\
\hline \multicolumn{7}{|l|}{$\begin{array}{l}\text { Antipsychotic side-effect } \\
\text { on metabolism }\end{array}$} \\
\hline Mild & $1.09(-2.04$ to 4.22$)$ & 0.49 & $-0.34(-1.20$ to 0.52$)$ & 0.43 & $0.28(-0.00$ to 0.56$)$ & 0.06 \\
\hline Strong & $2.73(-0.38$ to 5.83$)$ & 0.09 & $-0.05(-0.91$ to 0.81$)$ & 0.91 & 0.35 (0.07 to 0.63$)$ & 0.01 \\
\hline
\end{tabular}

circumference) in waist circumference after 12 months. In the control group this was $17.8 \%$ and a substantially higher $29.3 \%$, respectively $(P=0.075)$.

To investigate whether subgroups differed in their response to the intervention, stratified analyses were performed for gender, age

(a)

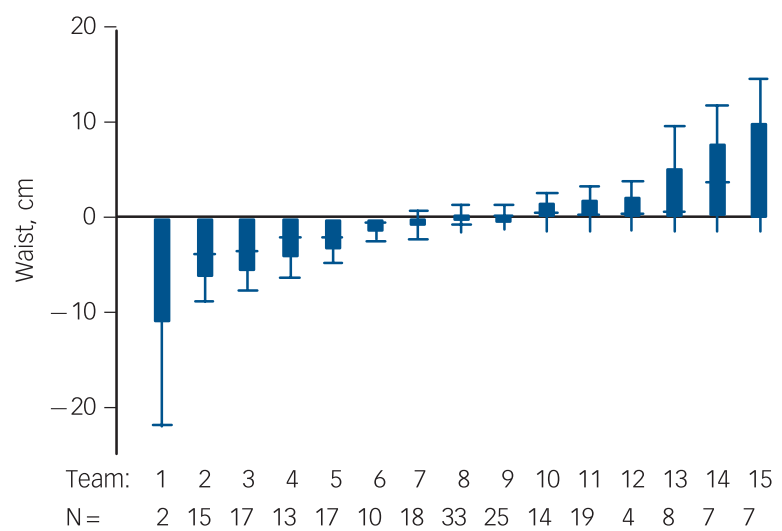

(c)

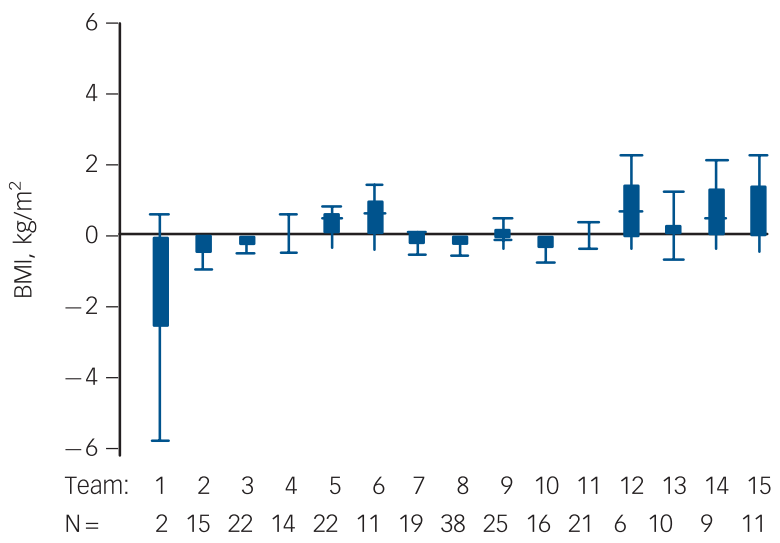

groups and housing facility. The intervention effect was most pronounced in males (waist circumference: $-2.42 \mathrm{~cm}(95 \% \mathrm{CI}$ -4.10 to -0.74$)$ and metabolic syndrome $z$-score: -0.33 s.d. $(95 \% \mathrm{CI}-0.55$ to -0.10$)$ ) and younger participants (metabolic syndrome $z$-score $\leqslant 43$ years: -0.31 s.d. $(95 \%$ CI -0.58 to

(b)

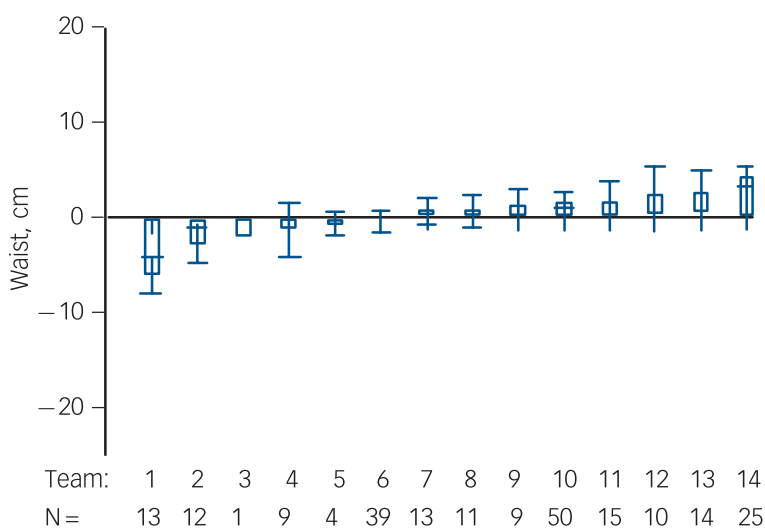

(d)

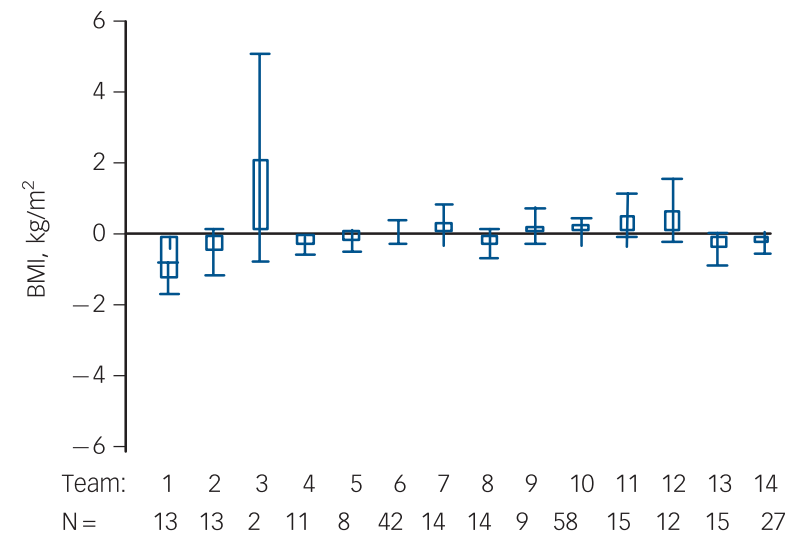

Fig. 2 Heterogeneity between teams in mean changes in waist circumference and body mass index (BMI) after 12 months.

Mean change in waist circumference $(\mathrm{cm})$ and $\mathrm{BMI}\left(\mathrm{kg} / \mathrm{m}^{2}\right)$ from baseline to 12 months post-baseline per team with $n$ patients. Waist change in the $(\mathrm{a})$ intervention and $(\mathrm{b})$ contro groups; BMI change in the (c) intervention and (d) control groups. Error bars represent standard errors of the mean. 
- 0.05) after 3 months (online Table DS2). The decrease in waist circumference and metabolic syndrome $z$-score in the intervention group was strongest in participants living in sheltered facilities, after 3 (waist circumference: $-1.68 \mathrm{~cm} \quad(95 \%$ CI -3.34 to $-0.01)$; metabolic syndrome $z$-score: -0.31 s.d. (95\% CI -0.51 to -0.11 )) and 12 months (waist circumference: $-2.63 \mathrm{~cm} ; 95 \%$ CI -4.28 to -0.98 ) whereas those in the intervention group in long-term clinical facilities showed a small increase in metabolic syndrome $z$-score $(0.25$ s.d. (95\% CI 0.00-0.49)) after 12 months.

For comparability with lifestyle intervention studies including patients with SMI with a BMI $\geqslant 25 \mathrm{~kg} / \mathrm{m}^{28}$, we performed sensitivity analyses in this subgroup. Findings remain the same: waist circumference was reduced by $1.79 \mathrm{~cm}(95 \% \mathrm{CI}-3.50$ to $-0.08)$ at 3 months and a trend for reduced waist circumference of $1.59 \mathrm{~cm}(95 \% \mathrm{CI}-3.34$ to 0.16$)$ was found at 12 months. Metabolic syndrome $z$-scores decreased by 0.20 s.d. (95\% CI -0.40 to -0.01$)$ after 3 months. Again, no intervention effects on BMI were found.

\section{Discussion}

\section{Main findings}

This large, multisite, RCT showed that changing the obesogenic environment of patients with SMI in residential settings into a healthier environment significantly reduced waist circumference and degree of metabolic syndrome after 3 months of intervention compared with care as usual. The magnitude of these effects decreased when, after 3 months, staff took over the lifestyle activities. This shows that improving the obesogenic environment can evoke beneficial changes without targeting patients directly, but sustainability remains a challenge.

An innovative feature of the ELIPS intervention is the focus on the obesogenic environment as opposed to directly and solely targeting patients' dietary and/or sedentary behaviours. ${ }^{13}$ Moreover, structural changes in the environment will affect every patient, irrespective of their personal interest in improving their lifestyles. Changing the environment using a small change approach indeed resulted in a small improvement: $1.5 \mathrm{~cm}$ in waist circumference over 3 months. If this can be sustained over a longer period of time, it will however, lead to clinically relevant improvements in adiposity and thereby reducing risk of cardiovascular disease and diabetes. ${ }^{28,29}$ Earlier studies found that for every $5 \mathrm{~cm}$ increase in waist circumference the risk of death increased $13 \%$ for females and $17 \%$ for males. ${ }^{30}$

\section{Comparison with previous studies}

Two earlier RCTs focused on residential patients, targeting both patients and staff with a 12-month lifestyle intervention with the structural guidance of external coaches. ${ }^{31,32}$ Forsberg and colleagues did not find improvements in waist circumference or glucose levels. ${ }^{31}$ This was, however, a small study $(n=41)$. Hjorth and colleagues showed a reduction of $3.1 \mathrm{~cm}$ in waist circumference and stabilised fasting glucose levels $v$. increased glucose levels in controls, which is comparable with our finding of reductions in waist circumference and fasting glucose $z$-score. The improvement in glucose metabolism is consistent with studies showing that lifestyle interventions are effective at preventing type 2 diabetes in the general population. ${ }^{33}$ The beneficial effect on fasting glucose suggests that lifestyle improvements, possibly via increased physical activity, improve insulin resistance. ${ }^{34}$ Changes in physical activity may result in changes in body composition with reduced fatness and increased muscle mass, ${ }^{32}$ which may explain the significant effects on waist circumference but not
BMI (Fig. 2). A meta-analysis of lifestyle interventions for SMI in both in- and out-patients confirmed our results on waist circumference (Cohen's $d=-0.37,95 \%$ CI -0.60 to -0.13 ) and fasting glucose (Cohen's $d=-0.24,95 \%$ CI -0.32 to $-0.10){ }^{6}$ Studies using individual and/or group counselling sessions, for example Daumit and colleagues ${ }^{8}$ and McKibbin et $a l,{ }^{35}$ found a significant decrease of $2.0-3.7 \mathrm{~cm}$ in waist circumference after 6 months of exercise, weight management or psychoeducational sessions. Thus, a lifestyle intervention in residential patients with SMI focusing on the obesogenic environment may yield results comparable to interventions targeting patients directly with individual or group counselling.

\section{Possible factors influencing intervention implementation and sustainability}

Changes in waist circumference varied widely between teams (Fig. 2). This is most likely related to the ease with which teams implemented and sustained new lifestyle habits. Structural aspects played a role, such as environmental features of the facility (for example physical activity opportunities in urban $v$. rural settings ${ }^{36}$ ), available budget (for example for healthy food products) and availability of staff members (for example nurses being scheduled to organise activities). Furthermore, logistic changes (for example altering the type of bread offered during lunch) were possibly more easily implemented than cultural changes (for example offering walk-and-talk therapy ${ }^{37}$ instead of sitting in the counselling office). Perhaps of greater influence were attitudes of staff: nurses differed in their experience of conflicting priorities (such as a high workload), conflicts with role definitions (for example nurses are not dieticians or physical therapists) and conflicts with own health behaviours (for example setting a good example by not ordering pizza during night shifts). ${ }^{38,39}$

The pragmatic character of the ELIPS trial allowed the intervention to be tailored to the resources of the facility. Moreover, regular staff implemented the intervention in everyday practice after 3 months of lifestyle coaching, giving a clear indication of what is attainable in 'real-world' settings. The design of the study, consisting of an implementation and a monitoring (support) phase, demonstrated the difficulty of sustaining achieved improvements. Despite involvement of regular staff in organising lifestyle activities and embedding lifestyle activities in teams' working routine, the magnitude of effects achieved at 3 months decreased in the 9 months thereafter, when staff members were less frequently guided by lifestyle coaches. This is in line with findings from a meta-analysis of lifestyle interventions by Bradshaw et al. ${ }^{39}$ and the study of Daumit and colleagues ${ }^{8}$ where initial significant effects on waist circumference were no longer significant when the frequency of sessions decreased and trained staff members took over most of the activities of lifestyle coaches. So, improvements in waist circumference and glucose levels are within reach, but sustainability might be achieved only when staff members are guided on a regular basis by a lifestyle coach whose primary responsibility is to promote the patients' lifestyle. The frequency of these guiding contacts needed to sustain or maximise results in the long term, should be explored, but likely needs to exceed two visits in 9 months.

The ELIPS intervention seemed to be especially beneficial for men and patients living in sheltered facilities. Perhaps the lifestyle activities, possibly the physical activities, were more appealing to male than female participants. Staff in long-term clinical care facilities might have experienced more obstacles in changing routines, anticipating dysregulation of the most severely ill patients. However, these results need to be interpreted with caution as subanalyses inevitably contained fewer patients than 
needed according to the power calculation, which was based on the comparison of intervention and control groups only.

\section{Strengths and limitations}

Strengths and limitations of the study are related to the pragmatic character of the RCT. The control condition was less controlled than it would have been in an explanatory trial. ${ }^{19}$ Despite being in the control condition, staff members or patients may have taken initiative to work on a more healthy lifestyle, following the trend in society. The intervention condition would have differed less between facilities if we had not used a team-tailored lifestyle plan. Using an implementation approach, however, largely increases the external validity of the study results. Our inclusion strategy further increased the external validity by avoiding selection bias of participating patients. ${ }^{19}$

\section{Implications}

A small change approach focusing on the obesogenic environment of patients living in sheltered or long-term care facilities has the potential to produce clinically relevant reductions in adiposity and thereby reduce cardiometabolic risk. However, our small results indicate that changing the obesogenic environment alone is not enough. It should be considered a prerequisite for improving patients' health ${ }^{13}$ and be part of an integrated approach of multiple targets, including sensible pharmaceutical strategies. A next step would be to develop a scalable (nursing) programme for maintenance of healthy changes and initiatives in the facilities, that is effective, affordable and sustainable in the long term. Anne Looijmans, MSc, Department of Epidemiology and Rob Giel Research Center,
University Medical Center Groningen, University of Groningen, Groningen;
Annemarie P. M. Stiekema, PhD, Department of Rehabilitation, Lentis Psychiatric
Institute, Zuidlaren; Richard Bruggeman, MD, PhD, Department of Psychiatry and
Rob Giel Research Center, University Medical Center Groningen, University of
Groningen, Groningen; Lisette van der Meer, PhD, Rob Giel Research Center,
University Medical Center Groningen, University of Groningen, Groningen, and
Department of Rehabilitation, Lentis Psychiatric Institute, Zuidlaren; Ronald P. Stolk,
MD, PhD, Department of Epidemiology, University Medical Center Groningen,
University of Groningen, Groningen; Robert A. Schoevers, MD, PhD, Department of
Psychiatry, University Medical Center Groningen, University of Groningen, Groningen;
Frederike Jörg, PhD, Rob Giel Research Center, University Medical Center
Groningen, University of Groningen, Groningen, and Research Department, Friesland
Mental Health Services, Leeuwarden; Eva Corpeleijn, PhD, Department of
Epidemiology and Rob Giel Research Center, University Medical Center Groningen,
University of Groningen, Groningen, The Netherlands

Correspondence: Anne Looijmans, MSc, University Medical Center Groningen Department of Epidemiology (FA40), PO Box 30.001, 9700 RB Groningen, The Netherlands. Email: A.Looijmans@umcg.n!

First received 30 Jan 2017, final revision 17 Mar 2017, accepted 22 Jul 2017

\section{Funding}

This work was supported by a grant from ZonMW (171101002). We received in-kind support from GGZ Friesland, Lentis and the Rob Giel Research Center.

\section{Acknowledgements}

We thank all patients, teams and organisations who participated in this trial. We thank Jacqueline Cambier, MSc, Human Movement Science and expert by experience, for her helpful remarks on the design of the study and advice in obtaining funding. We also thank helpful remarks on the design of the study and advice in obtaining funding. We also thank
Dr Ellen Visser for data management and all helpful instructions. Neither received any compensation for their contribution.

\section{References}

1 Laursen TM, Wahlbeck K, Hällgren J, Westman J, Ösby U, Alinaghizadeh H et al. Life expectancy and death by diseases of the circulatory system in patients with bipolar disorder or schizophrenia in the nordic countries. PLOS One 2013; 8: 1-7.
2 Zimmet P, Magliano D, Matsuzawa Y, Alberti G, Shaw J. The metabolic syndrome: a global public health problem and a new definition. J Atheroscler Thromb 2005; 12: 295-300

3 Hert M, Correll CU, Bobes J, Cetkovich-Bakmas M, Cohen D, Asai I, et al. Physical illness in patients with severe mental disorders. I. prevalence, impact of medications and disparities in health care. World Psychiatry 2011; 10: $52-77$.

4 McEvoy JP, Meyer JM, Goff DC, Nasrallah HA, Davis SM, Sullivan L, et al. Prevalence of the metabolic syndrome in patients with schizophrenia: Baseline results from the clinical antipsychotic trials of intervention effectiveness (CATIE) schizophrenia trial and comparison with national estimates from NHANES III. Schizophr Res 2005; 80: 19-32.

5 McGinty EE, Baller J, Azrin ST, Juliano-Bult D, Daumit GL. Interventions to address medical conditions and health-risk behaviors among persons with serious mental illness: a comprehensive review. Schizophr Bull 2016; 42 96-124.

6 Bruins J, Jörg F, Bruggeman R, Slooff C, Corpeleijn E, Pijnenborg M. The effects of lifestyle interventions on (long-term) weight management, cardiometabolic risk and depressive symptoms in people with psychotic disorders: a meta-analysis. PloS One 2014; 9: e112276.

7 Cabassa L, Ezell J, Lewis-Fernández R. Lifestyle interventions for adults with serious mental illness: a systematic literature review. Psychiatr Serv 2010; 61: $774-82$

8 Daumit GL, Dickerson FB, Wang N, Dalcin A, Jerome GJ, Anderson CAM, et al. A behavioral weight-loss intervention in persons with serious mental illness N Engl J Med 2013; 368: 1594-602.

9 Brar JS, Ganguli R, Pandina G, Turkoz I, Berry S, Mahmoud R. Effects of behavioral therapy on weight loss in overweight and obese patients with schizophrenia or schizoaffective disorder. J Clin Psychiatry 2005; 66: 205-12.

10 Alvarez-Jimenez M, Gonzalez-Blanch C, Vazquez-Barquero JL, Pérez-Iglesias R, Martínez-García O, Pérez-Pardal T, et al. Attenuation of antipsychoticinduced weight gain with early behavioral intervention in drug-naive first-episode psychosis patients: a randomized controlled trial. J Clin Psychiatry 2006; 67: 1253-60.

11 Wu R, Zhao J, Jin H, Shao P, Fang MS, Guo XF, et al. Lifestyle intervention and metformin for treatment of antipsychotic-induced weight gain: a randomized controlled trial. JAMA 2008; 299: 185-93.

12 Hassapidou M, Papadimitriou K, Athanasiadou N, Tokmakidou V, Pagkalos I, Vlahavas $\mathrm{G}$, et al. Changes in body weight, body composition and cardiovascular risk factors after long-term nutritional intervention in patients with severe mental illness: an observational study. BMC Psychiatry 2011; 11: 31-8.

13 Swinburn B, Egger G, Raza F. Dissecting obesogenic environments: the development and application of a framework for identifying and prioritizing environmental interventions for obesity. Prev Med 1999; 29: 563-70.

14 Faulkner G, Cohn TA. Pharmacologic and nonpharmacologic strategies for weight gain and metabolic disturbance in patients treated with antipsychotic medications. Can J Psychiatry 2006; 51: 502-11.

15 Cohn T, Grant S, Faulkner GE. Schizophrenia and obesity: addressing obesogenic environments in mental health settings. Schizophr Res 2010; 121: $277-8$.

16 Melamed Y, Stein-Reisner O, Gelkopf M, Levi G, Sivan T, Ilievici G, et al. Multi-modal weight control intervention for people with persistent mental disorders. Psychiatr Rehabil J 2008; 31: 194-200.

17 Sbrocco T, Nedegaard RC, Stone JM, Lewis EL. Behavioral choice treatment promotes continuing weight loss: preliminary results of a cognitive-behavioral decision-based treatment for obesity. J Consult Clin Psychol 1999; 67: 260

18 Looijmans A, Jörg F, Schoevers RA, Bruggeman R, Stolk RP, Corpeleijn E. Changing the obesogenic environment of severe mentally ill residential patients: ELIPS, a cluster randomised study design. BMC Psychiatry 2014; 14: 293.

19 Treweek S, Zwarenstein M. Making trials matter: pragmatic and explanatory trials and the problem of applicability. Trials 2009; 10: 9.

20 Bruins J, Pijnenborg MG, Bartels-Velthuis AA, Visser E, van den Heuvel ER, Bruggeman $\mathrm{R}$, et al. Cannabis use in people with severe mental illness: the association with physical and mental health - a cohort study. A pharmacotherapy monitoring and outcome survey study. J Psychopharmacol 2016; 30: 354-62.

21 Grundy SM, Cleeman JI, Daniels SR, Donato KA, Eckel RH, Franklin BA, et al. Diagnosis and management of the metabolic syndrome: An American Heart Association/National Heart, Lung, and Blood Institute scientific statement. Circulation 2005; 112: 2735-52.

22 Forouhi N, Balkau B, Borch-Johnsen K, Dekker J, Glumer C, Qiao Q, et al. The threshold for diagnosing impaired fasting glucose: a position statement by the European diabetes epidemiology group. Diabetologia 2006; 49: 822-7. 
23 International Expert Committee. International expert committee report on the role of the A1C assay in the diagnosis of diabetes. Diabetes Care 2009; 32: 1327-34.

24 Eisenmann JC. On the use of a continuous metabolic syndrome score in pediatric research. Cardiovasc Diabetol 2008; 7: 17-22.

25 Bakker SJ, Gansevoort RT, de Zeeuw D. Metabolic syndrome: a fata morgana? Nephrol Dial Transplant 2007; 22: 15-20.

26 Zorginstituut Nederland [National Health Care Institute]. Farmacotherapeutisch Kompas [Pharmacotherapeutic Compass]. Zorginstituut Nederland, 2015 (www.farmacotherapeutischkompas.nl/ inleidendeteksten/i/inl\%20antipsychotica.asp)

27 UpToDate. Selected Adverse Effects of Antipsychotic Medications for Schizophrenia. Wolters Kluwer, 2015 (www.uptodate.com/contents/ image? imageKey = PSYCH\%2F82533\&topicKey = PSYCH\%2F15766\&source =see_link\&utdPopup = true)

28 Farin HM, Abbasi F, Reaven GM. Comparison of body mass index versus waist circumference with the metabolic changes that increase the risk of cardiovascular disease in insulin-resistant individuals. Am J Cardiol 2006; 98 $1053-6$.

29 Fox KA, Despres JP, Richard AJ, Brette S, Deanfield JE, IDEA Steering Committee and National Co-ordinators. Does abdominal obesity have a similar impact on cardiovascular disease and diabetes? A study of 91,246 ambulant patients in 27 European countries. Eur Heart J 2009; 30: 3055-63.

30 Pischon $\mathrm{T}$, Boeing $\mathrm{H}$, Hoffmann $\mathrm{K}$, Bergmann $\mathrm{M}$, Schulze MB, Overvad $\mathrm{K}$, et al General and abdominal adiposity and risk of death in Europe. $N$ Engl J Med 2008; 359: 2105-20.
31 Forsberg KA, Björkman T, Sandman PO, Sandlund M. Physical health - a cluster randomized controlled lifestyle intervention among persons with a psychiatric disability and their staff. Nord J Psychiatry 2008; 62: 486-95.

32 Hjorth P, Davidsen AS, Kilian R, Pilgaard Eriksen S, Jensen SO, Sørensen HØ, et al. Improving the physical health of long-term psychiatric inpatients. Aust NZ J Psychiatry 2014; 48: 861-70.

33 Roumen $\mathrm{C}$, Blaak $\mathrm{EE}$, Corpeleijn $\mathrm{E}$. Lifestyle intervention for prevention of diabetes: Determinants of success for future implementation. Nutr Rev 2009; 67: $132-46$

34 Goodyear $\mathrm{P}$, Laurie J, Kahn M, Barbara B. Exercise, glucose transport, and insulin sensitivity. Annu Rev Med 1998; 49: 235-61.

35 McKibbin CL, Patterson TL, Norman G, Patrick K, Jin H, Roesch S, et al. A lifestyle intervention for older schizophrenia patients with diabetes mellitus: a randomized controlled trial. Schizophr Res 2006; 86: 36-44.

36 Kaczynski AT, Henderson KA. Environmental correlates of physical activity: a review of evidence about parks and recreation. Leis Sci 2007; 29: 315-54.

37 Doucette PA. Walk and talk: an intervention for behaviorally challenged youths. Adolescence 2004; 39: 373-88.

38 Lean $\mathrm{M}$, Leavey $\mathrm{G}$, Killaspy $\mathrm{H}$, Green $\mathrm{N}$, Harrison I, Cook $\mathrm{S}$, et al. Barriers to the sustainability of an intervention designed to improve patient engagement within NHS mental health rehabilitation units: a qualitative study nested within a randomised controlled trial. BMC Psychiatry 2015; 15: 209.

39 Bradshaw T, Wearden A, Marshall M, Warburton J, Husain N, Pedley R, et al. Developing a healthy living intervention for people with early psychosis using the medical research council's guidelines on complex interventions: Phase 1 of the HELPER-InterACT programme. Int J Nurs Stud 2012; 49: 398-406.

\section{poems by doctors}

\section{The Clearing}

\section{Daniel Racey}

Dawn after clerking overdoses,

in the night, we paused the pouring

of grief to let thunder pass

until we could hear

the rain fall and fall again.

I could have been a deer

on the edge of a clearing,

senses so keen I was trembling.

A soil loosed scent rushed

me and the sky was too big to pretend

The truth is I am as damned

and blessed as them, would be mad

to say other, though my notes

are a different telling.

In my night, I made a story

of that night and cried

that I catch this self so seldom,

Its bronze back dipping into the trees.

Somehow, I stitched the listening wound

and pulled myself up the stairs

to the blue light of your body.

(c) Daniel Racey, reproduced with permission.

The poem received a Commendation in the 2014 Hippocrates Prize for Poetry and Medicine. 